\title{
Comparing the Effectiveness of 3D Printing Technology in the Treatment of Clavicular Fracture between Surgeons with Different Experiences
}

\section{Jiang long Guo}

zhuhai houspital Guangdong Provincial Hospital of Traditional Chinese Medicine: The Second Affiliated Hospital of Guangzhou University of Chinese Medicine

Hong yi Li

zhuhai houspital Guangdong Provincial Hospital of Traditional Chinese Medicine: The Second Affiliated Hospital of Guangzhou University of Chinese Medicine

\section{Kui Zhao}

zhuhai houspital Guangdong Provincial Hospital of Traditional Chinese Medicine: The Second Affiliated Hospital of Guangzhou University of Chinese Medicine

\section{Meng Zhang}

zhuhai houspital Guangdong Provincial Hospital of Traditional Chinese Medicine: The Second Affiliated Hospital of Guangzhou University of Chinese Medicine

\section{Jing zhi Ye}

zhuhai houspital Guangdong Provincial Hospital of Traditional Chinese Medicine: The Second Affiliated Hospital of Guangzhou University of Chinese Medicine

\section{Jun Chen}

zhuhai houspital Guangdong Provincial Hospital of Traditional Chinese Medicine: The Second Affiliated Hospital of Guangzhou University of Chinese Medicine

\section{mengqiang xiao ( $\nabla$ xmqzhuhai@163.com )}

zhuhai houspital Guangdong Provincial Hospital of Traditional Chinese Medicine: The Second Affiliated Hospital of Guangzhou University of Chinese Medicine https://orcid.org/0000-0001-6970-6405

Jing feng Liu

zhuhai houspital Guangdong Provincial Hospital of Traditional Chinese Medicine: The Second Affiliated Hospital of Guangzhou University of Chinese Medicine

\section{Research article}

Keywords: COVID-19, Humidity, SARS-COV-2, Temperature, Weather

Posted Date: October 5th, 2021

DOI: https://doi.org/10.21203/rs.3.rs-940167/v1 
License: (c) (i) This work is licensed under a Creative Commons Attribution 4.0 International License. Read Full License 


\section{Abstract \\ Purpose}

To comparethe effectiveness of the three-dimensional (3D) printing technology in the treatment of clavicularfracturebetween experienced and inexperienced orthopedic surgeons.

\section{Methods}

A total of 80 patients with clavicle fracture (from February 2017 to May 2021)were enrolled in our study. Patients were divided randomly into four groups: group A: Patients underwent low-dose CT scan and 3D models were printed before surgeries performed by inexperienced surgeons; group B: Standard-dose CT were taken and 3D models were printed before surgeries performed by experienced surgeons; group $\mathrm{C}$ and D: Standard-dose CT were taken in both groups, and the operations were performed differently by inexperienced (group C) and experienced (group D) surgeons. Operation time, blood loss, length of incision and number of intraoperative fluoroscopy were recorded.

\section{Results}

No statistically significant differences were found in age, gender, fracture site and fracture type ( $P$ value: 0.23-0.88).Group A showed shorter incision length and less intraoperative fluoroscopy times than group $C$ and $D$ ( $P$ value $<0.05)$. There were no significant differences in blood loss volume, incision length and number of intraoperative time between group $A$ and group $B$ ( $P$ value range: $0.11-0.28$ ). The operation time of group $A$ was no longer than that of group $C$ and $D$ ( $P$ value range: 0.11 and 0.24$)$.

\section{Conclusion}

The surgical effectiveness of inexperienced surgeons who applied 3D printing technology before clavicular fracture operation were better than those of both inexperienced and experienced surgeons did not use preoperative 3D printing technology.

\section{Introduction}

Clavicle fracture is one of the common clinical diseases. Operative plate fixation is the main method for the treatment of clavicle fracture[1,2]. The clavicle is a slender $\mathrm{S}$ - shaped long bone with a superficial position and morphology varies greatly among individuals, which lead to a poor suitability of standard internal fixation. Therefore, using 3D printed model to assist internal fixation selection and surgical plan formulation seems important[3] . 
In recent years, with the popularity of 3D printing technology, more and more surgical plans were made by orthopedic surgeons based on conventional CT images and 3D printed models. Surgeons have recently begun to apply 3D printing technology in almost all areas of orthopedic trauma surgery[4,5]. By creating specific 3D printed anatomical models surgeons can better understand the anatomical structure details of patients' fractures, including trauma bone, surrounding soft tissue as well as normal areas. Thus 3D printing technology is helpful to make accurate preoperative plan [6-10].

The global unmet need for surgery, which is estimated to be over 320 million surgical procedures per year, is concentrated mostly in low- and middle-income countries due to lack of medical resources[11,12]. Less than half of the minimum needed procedures were done in low- and middle-income countries and one of the main reasons is surgeons with limited surgical skills [11-14].

In this study, we simulated the surgical effectiveness of preoperative 3D printing by inexperienced surgeons in low- and middle-income countries and the results were compared with those of surgeons with different experiences who only used preoperative CT evaluation.

\section{Materials And Methods 2.1 Study population}

This study was approved by the institutional review board of the Guangdong Hospital of traditional Chinese Medicine (BF2019-030-01).All patients with clavicle trauma admitted for preoperative imaging examination were included in this study (from February 2017 to May 2021). Inclusion criteria were as follows: age $\geq 18$ years, signed written consent, patients with suspected fractured shoulder from the emergency department. Exclusion criteria included: age $<18$ years, patients with conservative treatment, patients with pathological clavicle fractures, patients who rejected to provide written informed consent to participate in the study (Fig. 1). The study consisted of 80 patients (mean age 43.02 years; range 18-78 years), including 60 men and 20 women (Table 1).

80 inpatients were divided randomly into four groups (Fig. 1): group A (20 patients underwent low-dose CT scan and printed 3D fracture models before surgeries performed by inexperienced surgeons, which simulate the operations in low- and middle-income countries,), group B (20 patients underwent standarddose $C T$ and 3D models were printed before surgeries performed by experienced surgeons), group C (20 patients taken standard-dose CT and the operations were performed by inexperienced surgeons without 3D printed models) and group D (20 patients underwent standard-dose CT and the operations were performed by experienced surgeons without 3D printed models). The surgeons in group $A$ and $C$ were the same, while those in group B and D were the same. The operation time, blood loss (weighed gauze[15]), length of incision and number of intraoperative fluoroscopy were recorded in four groups.

\subsection{Image Acquisition And Production Of 3d Printed Model}


Patients with clavicular fracture were scanned by Toshiba 640-slice CT (Aquilion One; Canon Medical Systems, Otawara, Japan), and were randomly assigned to group A, B, C, and D (Fig. 1). In group A, the image quality simulated image quality of low-grade CT in basic hospital (low-grade Philips MX16-Slice CT in the Second People's Hospital of Xiangzhou District; tube voltage $120 \mathrm{kV}$, tube current $280 \mathrm{mAs}$ ). We retrospectively included 36 patients with clavicular fracture in the Second People's Hospital of Xiangzhou District and analyzed the image quality by giving a five-point Likert scale: $1=$ extremely poor; $2=$ poor; $3=$ fair; 4 = good and 5 = excellent. No significant difference of image quality score were found between group A and group in the Second People's Hospital of Xiangzhou District ( $4.40 \pm 0.60$ vs $4.36 \pm 0.55, p=$ 0.59). The CT scanning tube voltage is $120 \mathrm{kV}$ and the tube current is $150 \mathrm{mAs}$ in Group $B, C$ and $D$ (Table 2).

The PLA materials were used for 3D fracture model printing in group A and B. Digital models were generated from 3D-reconstructed CT data at 0.5-mm slice intervals using MICs Research software, version 19.0 and the skeleton model was printed by the melting deposition method on a CoLiDo 3.0 3Dprinter using white polylactic acid with a diameter of $1.75 \mathrm{~mm}$.

\subsection{Image Quality Evaluation}

To analyze the image quality of CT and 3D printed models, the double-blind five-pointLikert scale was used: excellent (5 points) : the quality of CT and 3D models was good, the surface of 3D models was smooth (fracture line and displacement of fracture was clearly shown), and it could be very helpful to make preoperation plan; good (4 points) : The surface of the 3D model is slightly blurred (the fracture line in the image is slightly blurred, and the displacement of the fracture is clear), it could be helpful to make preoperation plan; fair (3 points) : the surface of the 3D model is rough (the fracture line in the image is blurred, the artifact is obvious and the fracture displacement is clear), which basically does not affect the choice of surgical method and surgical treatment; poor(2 points) : quality of CT is poor and 3D model surface is slightly coarse (poor image quality shows that the fracture line, fracture displacement was not clearly shown), which affect on the choice of operation method and surgical treatment; extremely poor(1 point) : 3D model surface obviously coarse (image quality is poor), which was not helpful to choose surgical method.

One experienced surgeon as well as one inexperienced surgeon were randomly assigned to assess image quality in each patient. When the two surgeons have different opinions, they should negotiate and reach a consensus.

\subsection{Statistical Analysis}

Statistical analysis was performed using SPSS (IBM Corp, Armonk, NY, USA, version 26.0). One-way ANOVA and independent-samples $T$ test were used to determine if there were differences in patient age, operation time, blood loss, length of incision, number of intraoperative fluoroscopy and CT image quality 
score, 3D printing model score. ICC test was used to analyze the consistency of 3D model and image

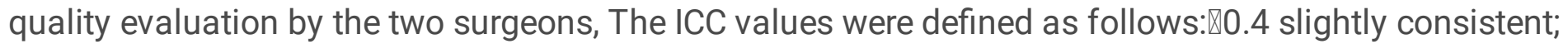
0.41 to 0.60 , moderately consistent; 0.61 to 0.80 , highly consistent; and 0.81 to 1.00 , almost perfectly consistent. Difference with $\mathrm{p}$ value区0.05 indicate statistical significance.

\section{Results}

No statistically significant differences were found in age, gender, fracture site and fracture type (Table 1 , $P$ value: $0.23-0.88$ ). The CT image quality score of group $A$ was $4.40 \pm 0.60$, which was less than that of the other three groups $(P<0.05)$. The CT image quality of one case in group $A$ was 3 points, and the rest were $\geq 4$ points, indicating good image quality. All cases of the other three groups were 5 points, indicating excellent image quality, which could meet the clinical needs. The image quality score of 3D printing models in four groups were as follows: only one case of 3D printing model in group A was evaluated as 4 points, while the other 3D printing models (in four groups) were evaluated as 5 points. ICC was 0.89 in group A for CT image quality. ICC was 0.99 for CT image quality evaluation and 3D printing model evaluation in group $B, C$ and $D$.

The operation time, blood loss, incision length and number of intraoperative fluoroscopy of each group are shown in Table 3. Group A showed shorter incision length and less intraoperative fluoroscopy times than group $C$ and $D$ ( $P$ value range: $0.01-0.01$ ). There were no significant differences in blood loss volume, incision length and number of intraoperative between group $A$ and group $B$ ( $P$ value range: $0.11-$ 0.28). The operative time of group $A$ was no longer than that of group $C$ and $D$ ( $P$ value: 0.11 and 0.24 ).

\section{Discussion}

This experiment simulated the preoperative image acquisition and surgical treatment of clavicle fracture in low- and middle-income countries. No difference was found comparing the image quality of high grade low-dose CT with that of low grade CT in our pre-experiment. In this study, low-dose CT scanning was performed in our hospital to simulate low-grade CT image quality in economically underdeveloped areas, and 3D models were printed. In order to simulate the surgeons with limited surgical skills in low- and middle-income countries, inexperienced surgeons in our hospital made surgical plans (group A) base on low-dose CT image and 3D printing models. Meanwhile, the operation time, blood loss, incision length and number of intraoperative fluoroscopy were recorded. No significant difference was found in operation time and blood loss between inexperienced doctors who use low-dose CT and 3D printing models (group A) and experienced surgeons use conventional CT (group D). However, the former group exhibited significantly shorter incision length and less intraoperative fluoroscopy time than the latter group. Based on the conventional CT images, surgical effect of experienced surgeons was better than inexperienced surgeons.

3D printing technology assisted the treatment of fracture internal fixation has good surgical effect, which has been confirmed in many studies[7-10,16,17]. In the past, the large size, expensive price of 3D printing 
machine and printing materials[17] made it difficult for 3D printing technology to be popularized in hospitals in low- and middle-income countries. Recently, 3D printing technology has been widely used in fracture surgery due to lower manufacturing costs, fewer printing steps and less complexity, which provides conditions for hospitals in low- and middle-income countries to use this technology. Standard home edition 3D printing machine was used in this study. The printing accuracy was $0.06-0.50 \mathrm{~mm}$, which could meet the needs of orthopedic surgery (Double Medical Internal fixation size $83.00-160.00 \mathrm{~mm}$, the size difference between adjacent models of the former was $4.00-12.00 \mathrm{~mm}$ ). The relatively low-cost material PLA (Tianwei Co., Ltd., US \$9.83 for 1KG PLA material) was used. Printing one 3D clavicle model costs about $\$ 0.84$ and takes about $3.01 \pm 0.20$ hours. Shuang $F$ et al. printed one elbow joint model for about $\$ 3.00$ which took about 4 hours[16]. In our study, 3D printing process self-study videos were provided by 3D printing manufacturers, and 3D modeling parameters were adjusted remotely by the manufacturers. Ordinary computers could run the software, which was easy to learn.

In the current study, many scholars studied the application of 3D printing technology to assist fracture surgery and achieved good surgical effects, however, none of them studied in groups according to surgeons' experience $[18,16,8,5,19]$. Our study compared the operation effectiveness of inexperienced and experienced surgeons using 3D printing technology and routine CT images before operations with that of same inexperienced and experienced surgeons only using routine CT scan before operations. The former has less incision length and less intraoperative exposure. Because 3D printing models could provide a visual, comprehensive vision of fracture, the position of plate implantation, screw direction and screw length can be determined in the simulation operation before operation. Therefore, when Group A simulated low-grade CT scan conditions in underdeveloped areas and with the assistance of 3D printing model, the operation time and blood loss of inexperienced surgeons were no different from those of inexperienced and experienced surgeons with conventional CT-assisted operations. 3D printing could be used as a supplement to routine CT scan, allowing surgeons to understand patients' fractures more intuitively and achieve better surgical results(Fig. 2).

The limitations of this study are as follows: 1 . This study only simulated the environment where hospitals equipped with low-grade CT and surgeons were inexperienced in economically underdeveloped areas, and did not really collect patients in economically underdeveloped areas. 2. The internal fixation involved in this study is not a customized internal fixation, but a relatively appropriate internal fixation selected from a series of sizes. 3. Due to the sample size of our study, we did not divided patients in subgroups using AO/OTA-2018 classification.

\section{Conclusion}

The surgical effectiveness of inexperienced surgeons who applied 3D printing technology before clavicular fracture operation were better than those of both inexperienced and experienced surgeons did not use preoperative 3D printing technology. The use of 3D models was beneficial for inexperienced surgeons making preoperative plan. 


\section{Declarations}

1. Acknowledgements

None.

2. Funding

The authors state that this work has not received any funding. The manuscript is truthful original work without fabrication, fraud or plagiarism.

3. Guarantor:

The scientific guarantor of this publication is Mengqiang, Xiao.

The e-mail address of the guarantor:xmqzhuhai@163.com

4. Conflict of Interest:

None.

5. Statistics and Biometry:

No complex statistical methods were necessary for this paper.

6. Informed Consent:

Written informed consent was obtained from all subjects (patients) in this study.

7. Ethical Approval:

Institutional Review Board approval was obtained.

8. Study subjects or cohorts overlap:

This study subjects have not been previously reported.

9. Methodology

Methodology: prospective randomised controlled trial

\section{References}

1. Yagnik GP, Porter DA, Jordan CJ (2018) Distal Clavicle Fracture Repair Using Cortical Button Fixation With Coracoclavicular Ligament Reconstruction. Arthrosc Tech 7 (4):e411-e415.

doi:10.1016/j.eats.2017.10.012 
2. Croley JS, Morris RP, Amin A, Lindsey RW, Gugala Z (2016) Biomechanical Comparison of Bicortical, Unicortical, and Unicortical Far-Cortex-Abutting Screw Fixations in Plated Comminuted Midshaft Clavicle Fractures. J Hand Surg Am 41 (6):703-711. doi:10.1016/j.jhsa.2016.04.001

3. Cronskär M, Rännar L-E, Bäckström M, Nilsson KG, Samuelsson B (2015) Patient-Specific Clavicle Reconstruction Using Digital Design and Additive Manufacturing. Journal of Mechanical Design 137 (11). doi:10.1115/1.4030992

4. Aimar A, Palermo A, Innocenti B (2019) The Role of 3D Printing in Medical Applications: A State of the Art. J Healthc Eng 2019:5340616. doi:10.1155/2019/5340616

5. Kumar Gupta D, Ali MH, Ali A, Jain P, Anwer MK, lqbal Z, Mirza MA (2021) 3D printing technology in healthcare: applications, regulatory understanding, IP repository and clinical trial status. J Drug Target:120. doi:10.1080/1061186X.2021.1935973

6. Ganguli A, Pagan-Diaz GJ, Grant L, Cvetkovic C, Bramlet M, Vozenilek J, Kesavadas T, Bashir R (2018) 3D printing for preoperative planning and surgical training: a review. Biomed Microdevices 20 (3):65. doi:10.1007/s10544-018-0301-9

7. Chen C, Cai L, Zheng W, Wang J, Guo X, Chen H (2019) The efficacy of using 3D printing models in the treatment of fractures: a randomised clinical trial. BMC Musculoskelet Disord 20 (1):65. doi:10.1186/s12891-019-2448-9

8. Xiao M, Zhang M, Lei M, Hu X, Wang Q, Chen Y, Ye J, Xu R, Chen J (2021) Application of ultra-low-dose CT in 3D printing of distal radial fractures. Eur J Radiol 135:109488. doi:10.1016/j.ejrad.2020.109488

9. Garg B, Gupta M, Singh M, Kalyanasundaram D (2019) Outcome and safety analysis of 3D-printed patient-specific pedicle screw jigs for complex spinal deformities: a comparative study. Spine J 19 (1):5664. doi:10.1016/j.spinee.2018.05.001

10. Kang HJ, Kim BS, Kim SM, Kim YM, Kim HN, Park JY, Cho JH, Choi Y (2019) Can Preoperative 3D Printing Change Surgeon's Operative Plan for Distal Tibia Fracture? Biomed Res Int 2019:7059413. doi:10.1155/2019/7059413

11. Glasziou P, Straus S, Brownlee S, Trevena L, Dans L, Guyatt G, Elshaug AG, Janett R, Saini V (2017) Evidence for underuse of effective medical services around the world. Lancet 390 (10090):169-177. doi:10.1016/S0140-6736(16)30946-1

12. Weiser TG, Haynes AB, Molina G, Lipsitz SR, Esquivel MM, Uribe-Leitz T, Fu R, Azad T, Chao TE, Berry WR, Gawande AA (2015) Estimate of the global volume of surgery in 2012: an assessment supporting improved health outcomes. Lancet 385 Suppl 2:S11. doi:10.1016/S0140-6736(15)60806-6

13. Guindon GE, Lavis JN, Becerra-Posada F, Malek-Afzali H, Shi G, Yesudian CA, Hoffman SJ, Research to P, Practice Study T (2010) Bridging the gaps between research, policy and practice in low- and middle- 
income countries: a survey of health care providers. CMAJ 182 (9):E362-372. doi:10.1503/cmaj.081165

14. Elliott IS, Sonshine DB, Akhavan S, Slade Shantz A, Caldwell A, Slade Shantz J, Gosselin RA, Coughlin RR (2015) What factors influence the production of orthopaedic research in East Africa? A qualitative analysis of interviews. Clin Orthop Relat Res 473 (6):2120-2130. doi:10.1007/s11999-015-4254-5

15. Awobusuyi JO, Mapayi FA, Adedolapo A (2008) Blood loss during vascular access cannulation: quantification using the weighed gauze and drape method. Hemodial Int 12 (1):90-93. doi:10.1111/j.1542-4758.2008.00247.x

16. Yang L, Grottkau B, He Z, Ye C (2017) Three dimensional printing technology and materials for treatment of elbow fractures. Int Orthop 41 (11):2381-2387. doi:10.1007/s00264-017-3627-7

17. Michielsen M, Van Haver A, Vanhees M, van Riet R, Verstreken F (2019) Use of three-dimensional technology for complications of upper limb fracture treatment. EFORT Open Rev 4 (6):302-312. doi:10.1302/2058-5241.4.180074

18. Weidert S, Andress S, Linhart C, Suero EM, Greiner A, Bocker W, Kammerlander C, Becker CA (2020) 3D printing method for next-day acetabular fracture surgery using a surface filtering pipeline: feasibility and 1-year clinical results. Int J Comput Assist Radiol Surg 15 (3):565-575. doi:10.1007/s11548-019-02110-0

19. Costan VV, Nicolau A, Sulea D, Ciofu ML, Boisteanu O, Popescu E (2021) The Impact of 3D Technology in Optimizing Midface Fracture Treatment-Focus on the Zygomatic Bone. J Oral Maxillofac Surg 79 (4):880-891. doi:10.1016/j.joms.2020.11.004

\section{Tables}

Table 1

Patients Demographic and Baseline Characteristics

\begin{tabular}{|llllll|}
\hline group & A & B & D & C & P \\
\hline No & 20 & 20 & 20 & 20 & \\
\hline Age & $45.50 \pm 13.41$ & $37.40 \pm 15.68$ & $44.1 \pm 10.75$ & $44.55 \pm 13.65$ & 0.42 \\
\hline Gender & $14 \mathrm{M} 6 \mathrm{~F}$ & $17 \mathrm{M} 3 \mathrm{~F}$ & $17 \mathrm{M} 3 \mathrm{~F}$ & $12 \mathrm{M} 8 \mathrm{~F}$ & 0.23 \\
\hline $\begin{array}{l}\text { Fracture site } \\
\text { (middle /Distal,case) }\end{array}$ & $15 / 5$ & $17 / 3$ & $18 / 2$ & $18 / 2$ & 0.65 \\
\hline Comminuted fracture(case) & 17 & 15 & 17 & 17 & 0.88 \\
\hline
\end{tabular}


Table 2

CT Parameters of each group

\begin{tabular}{|c|c|c|c|}
\hline Dose group & Standard & Ultra-low & $\mathbf{P}$ \\
\hline Tubevoltage (kV) & 120 & 100 & \\
\hline Tubecurrent (mA) & 150 & 140 & \\
\hline $\mathrm{D}-\mathrm{FOV}(\mathrm{mm})$ & 500 & 500 & \\
\hline Rotation time (s) & 1.00 & 0.75 & \\
\hline Thickness (mm) & 0.50 & 0.50 & \\
\hline Intrval(mm) & 0.50 & 0.50 & \\
\hline Scan length $(\mathrm{cm})$ & 160.00 & 160.00 & \\
\hline AIRD3D & Standard EU10 & Standard EU10 & \\
\hline CTDIvol(mGy) & $14.57 \pm 0.60$ & $6.67 \pm 0.25$ & 0.01 \\
\hline $\mathrm{ED}(\mathrm{mSv})$ & $2.17 \pm 0.36$ & $1.10 \pm 0.13$ & 0.01 \\
\hline
\end{tabular}

Table 3

Surgical effect and image quality were evaluated in each group

\begin{tabular}{|llllll|}
\hline Dose group & A & B & C & D & P \\
\hline operation time (min) & $103.65 \pm$ & $83.60 \pm$ & $117.50 \pm$ & $102.50 \pm$ & 0.01 \\
& 26.94 & 19.30 & 26.75 & 25.31 & \\
\hline blood loss (ml) & $24.00 \pm$ & $17.25 \pm$ & $31.50 \pm$ & $27.00 \pm$ & 0.01 \\
& 14.74 & 10.82 & 21.34 & 10.81 & \\
\hline Length of incision(cm) & $6.36 \pm 2.37$ & $7.25 \pm$ & $9.80 \pm 1.61$ & $9.15 \pm 1.84$ & 0.01 \\
& & 1.97 & & & \\
\hline number of Intraoperative & $5.95 \pm 1.05$ & $5.25 \pm$ & $13.85 \pm$ & $9.25 \pm 2.27$ & 0.01 \\
fluoroscopy (n) & & 0.97 & 3.05 & & \\
\hline $\begin{array}{l}\text { CT image quality assessment } \\
\text { (fraction) }\end{array}$ & $4.40 \pm 0.60$ & $5.00 \pm$ & $5.00 \pm 0.01$ & $5.00 \pm 0.01$ & 0.01 \\
\hline 3D fracture model(fraction) & $4.95 \pm$ & 5.01 & & & \\
& 0.224 & 0.01 & $5.00 \pm 0.01$ & $5.00 \pm 0.01$ & 0.30 \\
\hline
\end{tabular}

Figures 


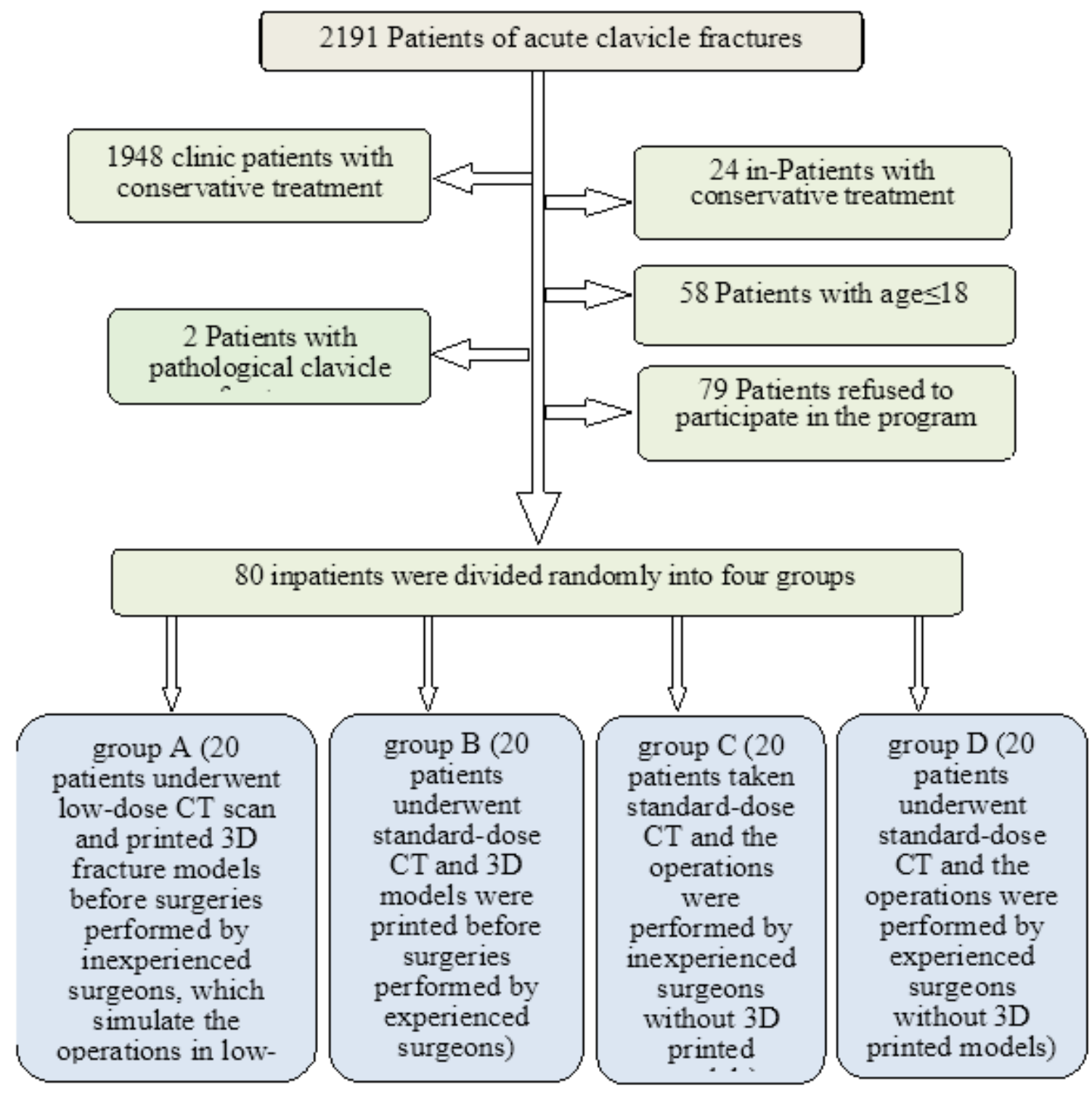

\section{Figure 1}

Flowchart of the study. 


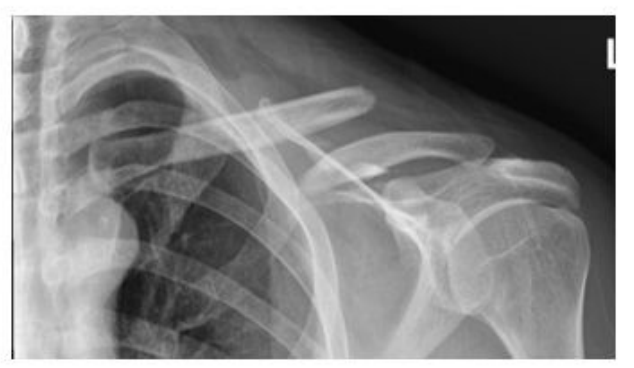

$2 \mathrm{a}$

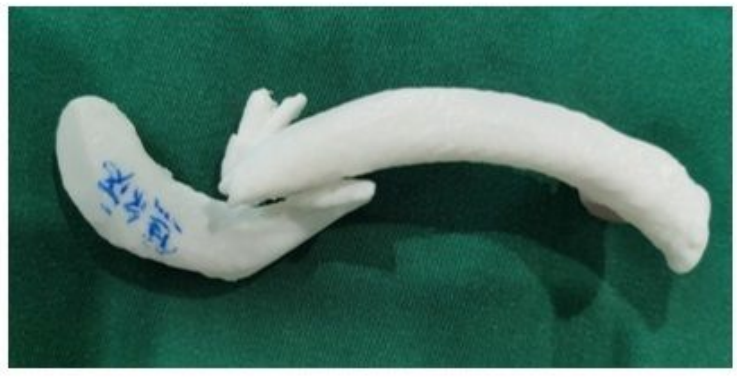

$2 \mathrm{c}$
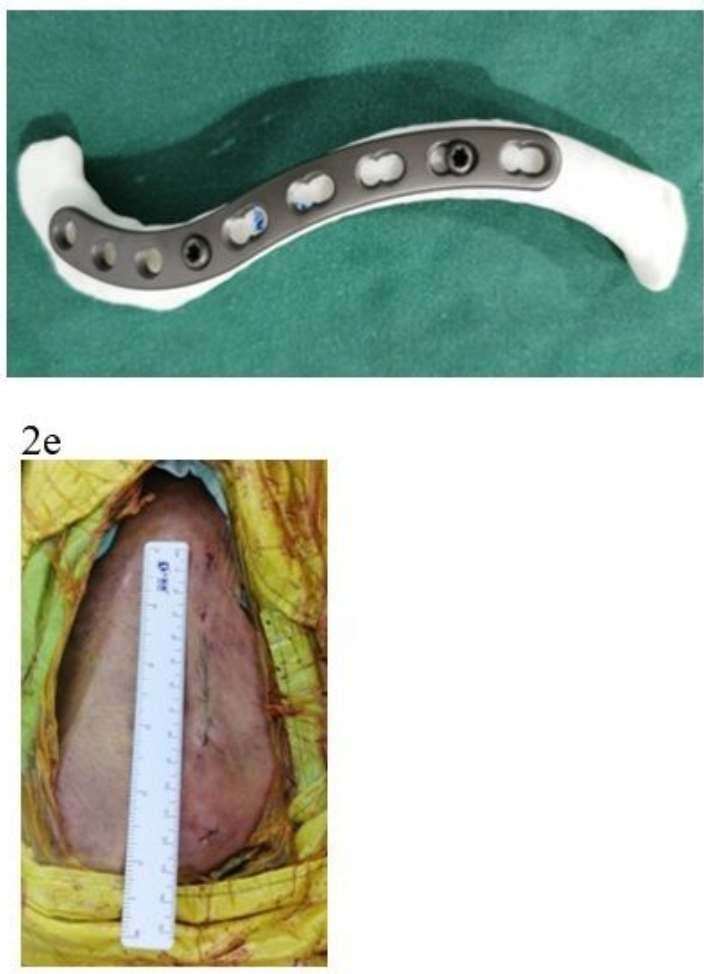

$2 \mathrm{~g}$

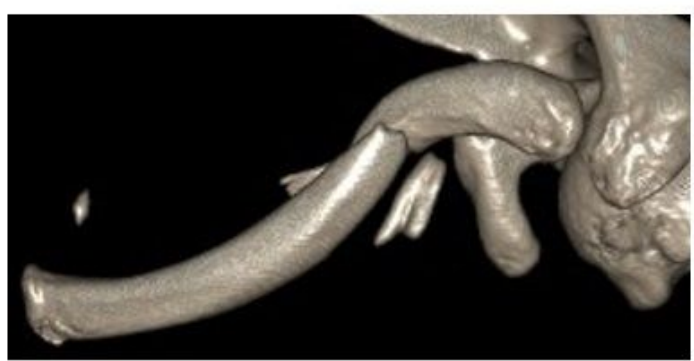

$2 b$

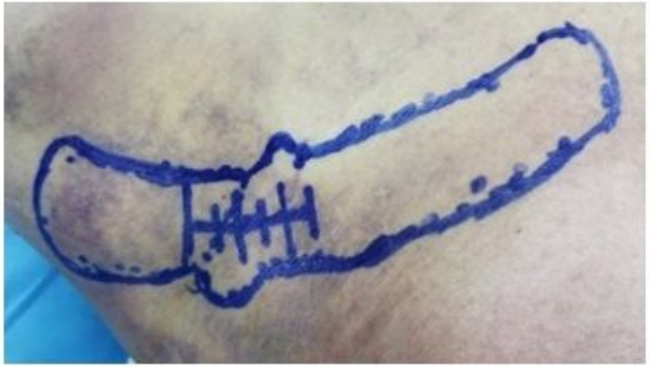

$2 d$

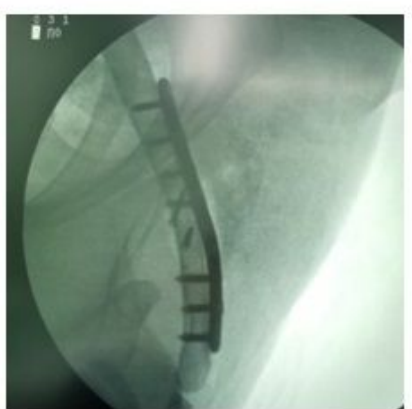

$2 \mathrm{f}$

\section{Figure 2}

A 53 years old male with middle site clavicle fracture. The operation time was about 100 minutes and the incision length was $8.30 \mathrm{~cm}$. During the operation, the blood loss was 10 milliliter and intraoperative fluoroscopy was performed five times. (2a) DR image; (2b) CT 3D-reconstruction; (2c) 3D printing model of clavicle fracture; (2d) preoperative marking; (2e) The internal fixation selection and preoperative 
delineation of clavicle fracture site; (2f) Intraoperative fluoroscopy of clavicle fracture; $(2 \mathrm{~g})$ incision length $(8.30 \mathrm{~cm})$. 